\title{
A Systematic Review of Blockchain Technology and Its Applications
}

\author{
Bharati H Naikwadi a ${ }^{1}$, K. G. Kharade ${ }^{\text {b }, ~ S . ~ Y u v a r a j ~}{ }^{\text {c }}$, K. Vengatesan ${ }^{\mathrm{d}}$ \\ ${ }^{a}$ BLDEA's A.S.Patil College of Commerce, Vijayapur, KA, India \\ ${ }^{b}$ Department of Computer Science, Shivaji University, Kolhapur, MH, India \\ ${ }^{c}$ Department of Electronics and Communication Engineering, SRM Institute of Science and Technology, \\ Kattankulathur, TN, India \\ ${ }^{d}$ Computer Engineering, Sanjivani College of Engineering, Kopargaon, MH, India
}

\begin{abstract}
Blockchain technology could radically transform our business environment in the decades to come. It has the power to redefine our views of business processes and may even impact our overall economy. The works comprehensively document the implementation of blockchain applications in various sectors. Our goal is to determine what state blockchain technology is in and what kinds of applications it has. A large majority of the current blockchainrelated research is dedicated to crypto currencies, such as Bitcoin. Only a small percentage of work is dedicated to exploring the wide range of potential blockchain technology applications. Blockchain technology has multiple applications across multiple industries. This study attempts to determine the opportunities and threats presented by Blockchain Technology for current or future applications. The number of published studies that were studied carefully and critically and added to the Block chain's body of knowledge was large.
\end{abstract}

Keywords. Applications of Blockchain, Blockchain, Electronic Health Records, IoT, Security

\section{Introduction}

Blockchains disrupted traditional business processes by introducing decentralized applications and transactions, which had previously relied on centralized architectures or trusted third parties to verify them. Blockchain technology provides characteristics like transparency, robustness, suitability, and security, all of which are inherent to the system [3]. Despite the revolutionary possibilities for building the future Internet system via blockchain technology, many technical challenges are currently confronted. The first issue is scalability. At present, the maximum block size in Bitcoin is one megabyte, and blocks are mined about every ten minutes. The system then limits the number of transactions the network can perform per second to seven, unable to deal with high-frequency trading [23]. In a public ledger, each transaction is verified by the agreement of most participants in the system. In other words, once data is entered, it can never be erased. Every transaction ever made is included in the Blockchain's immutable record [17]. An easy analogy to use is to say that it is easier to steal a cookie from a cookie jar, where the cookie jar is hidden away in a secluded location rather than to steal a cookie from a cookie jar, where there are numerous people around to witness the theft [5].

\footnotetext{
${ }^{1}$ K.Vengatesan,Computer Engineering, Sanjivani College of Engineering, Kopargaon, India Email: vengicse2005@gmail.com.
} 
Distributed ledger technology is commonly referred to as blockchain technology. The Blockchain is the underlying technology that makes bitcoin possible, devised by an anonymous individual known as Satoshi Nakamoto in 2008. It is best to understand how the bitcoin mechanism works before learning about Blockchain. Bitcoin's digital currency is a peer-to-peer, distributed, and decentralized payment system [1]. Like distributed ledger technology (DLT), Blockchain does not require a central authority to manage operations. No one knows who controls it, except for those directly involved. Blockchain data is organized in terms of blocks, which is why it is called a blockchain. This complex network of interlocked blocks is protected with encryption. The data added to the Blockchain is immutable, i.e., once added, it cannot be altered or deleted. It is one of the distinguishing features of this database compared to conventional databases. Every transaction exists in history because you cannot remove or alter even a single block of data [2].

\section{Literature Review}

(Xu et.al ,2019) identified that, many people see blockchain as a core technology that will disrupt an industry. A great deal of work has been done in the advancement of blockchain, but the amount of investigation remains limited. in order to thoroughly research block chain's current academic research, it incorporates the topics of business and economics [24]. (Junyao Wang et.al, 2019) focused on Blockchain development and application are being explored in both academic and commercial environments. Blockchain is already proving itself in various fields, particularly in the financial, sales, medical, and other fields [25]. (Kitsantas, 2019) revealed that, at the beginning of the blockchain era, where the technology will lead to significant changes in business environment, and that will have a profound impact on the decades to come. It can have a radical impact on the way we understand business processes, and can fundamentally transform our economy. The main purpose of blockchain technology is to provide transparency, data security, and integrity. This is because blockchain technology is impervious to tampering or fabrication [26]. (Liang Zhou, 2020) stated that, blockchain has recently emerged as a distributed and fast-evolving technology, and this has led to it influencing crypto currency and e-commerce, which have drawn the interests of governments, businesses, and research institutions. This suggests that institutions should plan their research effectively in order to get a sense of the current state of blockchain research [27].

\section{Research Methodology}

The present study employs a systematic literature review. A systematic literature review aims to find previous research relevant to the topic and then outline research gaps and future research paths. It also focuses on the benefits and applications of Blockchain and the areas where Blockchain has been implemented. During this research we have worked on areas where block chain can be implemented. More than 100 papers have been studied during this paper. It is observed that, block chain technology has many advantages but lack of awareness and government policies affecting on the use of this technology. Health care is the most common area where this technology is being implemented. With the rapid growth in all the industry, block chain 
is being used by many industries. We have identified few industries where it is being used.

\section{Benefits of Blockchain}

Block chain technology has various benefits; some of the benefits are;

a) Better Transparency: With the advancement of blockchain technology, data and money transactions are becoming easier. All participants share the document in the networks instead of being distributed to each of them. Even if the participants wanted to change the documentation, they would not do so without changing the documentation blocks that came before. It is because, in the context of heavy processes, blockchain technology has proven to be more accurate, consistent, and transparent [21]

b) Enhanced Security: Blockchain technology is superior to other platforms or record-keeping systems when it comes to security. All transactions, even the smallest ones, need to be approved through consensus. The process of generating and applying a cryptographic hash function, then making that hash transaction-based linked to the prior transaction, occurs every time a transaction is performed [13]. Network security is also strengthened because each node keeps a record of all the transactions. Thus, malicious actors would be unable to alter transactions since others in the network will reject their attempts to do so [8].

c) Reduced Costs: For the vast majority of businesses, cost reduction is of prime importance. Because Blockchain eliminates the need for third parties and mediators, you don't need to trust your trading partner. A single, immutable version will be available to everyone, so you will not have to review much documentation [6]

d) Data Ownership: Blockchain provides this vital benefit. Blockchain is widely distributed instead of being controlled from a single point. Thus, even if a third party or a government were to attempt to control their plan and your process, they could not succeed. Blockchain is simply a more descriptive term for a fair distribution system [18].

e) Accurate Traceability: The buyer is already familiar with how difficult it is to trace a product back to its source because you deal with traded products through a complex supply chain. You end up with an audit trail showing where each asset started its journey and where it stopped on the way. The transaction data from this historic trade could be used to verify the authenticity of support and help prevent [9]. 
f) Improved Speed and Highly Efficient: Trading anything is a timeconsuming process prone to human error and often requires third-party mediation, regardless of whether it's done through paper-heavy or digital means. The main benefit of using a single ledger shared by participants is that there is no need to reconcile multiple ledgers. As a result, you don't end up with as much clutter [10].

\section{Advantages of Blockchain Technology}

Blockchain technology has many advantages over traditional technologies.

$\checkmark$ You can trust your business process's quality because it is protected with a high level of security thanks to Blockchain.

$\checkmark$ Transactions done are transparent, which allows them to be tracked easily

$\checkmark$ Enterprise blockchain technology gives organizations the ability to control accessibility using a wide range of accessibility levels [11].

$\checkmark$ When organizations use Blockchain, they can perform transactions up to five times faster.

$\checkmark$ The threats to your business will be more significantly reduced because of hacking.

$\checkmark$ Blockchain has eliminated the need to pay for centralized entities or intermediaries' services, as the network is decentralized

$\checkmark$ Automated account reconciliation is possible [12].

\section{Applications of Blockchain for Businesses}

Typical applications of the Blockchain technology are;

a) Blockchain for Communications: The connectivity, infrastructures, resources, end devices, and applications in communications and networking systems are becoming much more complex and heterogeneous due to the rapid advancement of information and communication technologies [15]. Additionally, there is a significant volume of data and a large number of end devices that will bring substantial security, privacy, services, and network management complications. The combined consideration of Blockchain and machine learning (ML) may lead to significant benefits and have sparked a great deal of interest in both academia and industry [16]

b) Blockchain in Music Industry: A longstanding problem has increased in urgency: a central database that catalogs music metadata does not exist, making it challenging to find the owners or those with rights and ensure they get paid. A decentralized ledger, an unalterable, secure, efficient, and costeffective rights management system, could be built in the music industry; GRD, the centralized Global Repertoire Database, has failed so far [4].

c) Blockchain in IoT: For an IoT solution to succeed, it must possess reliability, scalability, and long-term durability. And we know IoT is useful. In contrast to Blockchain, Blockchain is much more experimental and episodic. It has not 
been scientifically proven. Blockchain flares up but then quickly levels off. Meanwhile, IoT solutions are continuously put into use [19]

d) Blockchain Empowered Cloud Gaming System: Cloud gaming offers cloud-rendered game scenes and real-time video footage delivered to end devices via the Internet. It allows players to access game services anytime and anywhere, even if their hardware capabilities are limited. Still, since the cloud gaming market is a commercial service, the latest payment models for cloud gaming are in the early development stages [22].

e) Blockchain in Healthcare: This idea comes out of the desire for healthcare security and interoperability. Much medical data is collected and transmitted daily thanks to IoT and the increasing abundance of health devices and mobile healthcare applications. This data traffic requires privacy and security management. With blockchain technology, it is possible to secure the recording and sharing of medical records while assuring each patient's data privacy. Furthermore, Blockchain has the advantage of making healthcare management more straightforward, but it also presents challenges that should be anticipated [14].

f) Blockchain In Energy Sector: For governments, one of the most critical aspects of energy distribution, allocation, and production has always been present. In the absence of a well-thought-out energy strategy, no government can provide necessary growth to its economy. Similarly, private players also contribute to the Blockchain, both materially and immaterially.

g) Blockchain in Banking: A Corda-based implementation is created through a merger of financial institutions and adheres to industry standards. Corda is a blockchain-based ledger platform, which has been appropriated. Hyper ledger Fabric blockchain platforms are widely accepted as secure and private blockchains. Banks find security assurance helpful. Ethereum is a type of platform that enables people to build decentralized applications [20].

h) Blockchain in Real Estate: For the past few years, the real estate sector's conditions have been subpar. Since house prices have increased and it has become more challenging to buy a property that is one reason. With the introduction of Blockchain, the real estate sector is looking at its operations in a whole new light. Blockchain will allow tokens to be issued in connection with particular actions. In other words, properties can be leased out using predefined programming. Also, tokens enable businesses to add any necessary business logic, such as combat fraud [7].

\section{Conclusion}

This paper demonstrated how blockchain technology is being applied in various industries, including finance and healthcare. While the first blockchain research was carried out in 2012, more research was conducted on the subject in the years since. This paper studies how blockchain technology can encourage open science, including reviewing the latest developments and identifying and describing the science's advantages and challenges. To test whether the requirements for an open scientific 
ecosystem fit with blockchain technology properties, we first identified them and compared them to see if they matched. Finally, we proved that the technology was reliable and appropriate for open science.

\section{References}

[1] Alla, S., \&Soltanisehat, L. (2018, May). Blockchain Technology in Electronic Healthcare System. https://www.researchgate.net/publication/325542378_Blockchain_Technology_in_Electronic_Healthca re_System

[2] Anwar, H. (2019, January 6). Blockchain vs. Distributed Ledger Technology: A Detailed Guide. https://101blockchains.com/blockchain-vs-distributed-ledger-technology/\#prettyPhoto

[3] Casino, F., Dasaklis, T. K., \&Patsakis, C. (2019). A systematic literature review of blockchain-based applications: Current status, classification, and open issues. Telematics and Informatics, 36, 55-81. https://doi.org/10.1016/j.tele.2018.11.006

[4] Celebucki, P., Atkins, T., Gutierrez, J., McKone, M., \& Steinberg, A. (n.d.). Blockchain + the Music Industry. Retrieved February 23, 2021, from https://onprem.com/wpcontent/uploads/2020/08/wp_blockchain-1.pdf

[5] Crosby, M., Nachiappan, Pradhan, P., Varma, S., \&Kalyanaraman, V. (2015). Sutardja Center for Entrepreneurship \& Technology Technical Report (p. 35). chromeextension://oemmndcbldboiebfnladdacbdfmadadm/https://scet.berkeley.edu/wpcontent/uploads/BlockchainPaper.pdf

[6] Dongale, T. D., Kharade, K. G., Naik, G. M., \& Kamat, R. K. (2017). Artificial Neural Network Modeling of NixMnxOx based Thermistor for Predicative Synthesis and Characterization. Journal of Nano And Electronic Physics, 03042(1)-03042(4).

[7] Geroni, D. (2021, January 27). Top 5 Benefits of Blockchain Technology. https://101blockchains.com/benefits-of-blockchain-technology/

[8] Hooper, M. (2018, February 22). Top five blockchain benefits transforming your industry_Blockchain Pulse: IBM Blockchain Blog. https:/www.ibm.com/blogs/blockchain/2018/02/top-five-blockchainbenefits-transforming-your-industry/

[9] Kharade, K. G., Kharade, S. K., \& Kumbhar, V. S. (2018). Impact of Digital India on Various Sectors. Indian Journal of Innovation in Management and Excellence In Research (IJIMER), 37-40.

[10] Kharade, K. G., Kharade, S. K., \& Oza, K. S. (2018). Security Threats In E-commerce. Indian Journal of Innovation In Management And Excellence In Research (IJIMER), 14-17.

[11] Kharade, S. K., Kamat, R. K., \& Kharade, K. G. (2019). Simulation of Dye Synthesized Solar Cell using Artificial Neural Network. International Journal of Engineering and Advanced Technology (IJEAT), 1316-1322.

[12] Kharade, K. G., Kamat, R. K., Kharade, S. K., \& Katkar, S. V. (2019). Automation of Paper Setting Process to Improve Effectiveness of The Examination System of The University. Journal of Emerging Technologies and Innovative Research, 490-493.

[13] Kharade, K. G., Kumbhar, V. S., \& Kharade, S. K. (2017). A Comparative Study of Traditional Server and Azure Server. Journal of Advances in Science and Technology (JAST), 329 - 331.

[14] Kharade, K. G., Mudholakr, R. R., Kamat, R. K., \& Kharade, S. K. (2019). Perovskite Solar Cell Simulation using Artificial Neural Network. International Journal of Emerging Technologies and Innovative Research, 336-340.

[15] Kharade, K. G., Mudholkar, R. R., Kamat, R. K., \& Kharade, S. K. (2019). Artificial Neural Network Modeling of CZTS Solar Cell For Predicative Synthesis And Characterization. International Journal of Research And Analytical Reviews, 997-1006.

[16] Potharaju, S. P., \& Sreedevi, M. (2017). A Novel Clustering Based Candidate Feature Selection Framework Using Correlation Coefficient for Improving Classification Performance. Journal of Engineering Science \& Technology Review, 10(6).

[17] Patil, B. P., Kharade, K. G., \& Kamat, R. K. (2020). Investigation on Data Security Threats \& Solutions. International Journal of Innovative Science and Research Technology, 5(1), 79-83.

[18] Rao, G. N., Kumari, K. A., Shankar, D. R., \& Kharade, K. G. (2021). A comparative study of augmented reality-based head-worn display devices. Materials Today: Proceedings.

[19] Schaefer, J. (2019, August 2). Blockchain Applications in IoT: Good and Bad | IoT For All. https://www.iotforall.com/blockchain-applications-in-iot

[20] Sharma, D. (2020). Application of Blockchain in an Indian Banking Sector. Global Scientific Journal, $8(5), 526-530$. 
[21] Techfunnel. (2020, May 13). 14 Amazing Benefits of Blockchain Technology | Techfunnel Guide. https://www.techfunnel.com/information-technology/benefits-blockchain-technology/

[22] Zhao, J., Chi, Y., Wang, Z., Leung, V. C. M., \& Cai, W. (2020). CloudArcade: A Blockchain Empowered Cloud Gaming System. Proceedings of the 2nd ACM International Symposium on Blockchain and Secure Critical Infrastructure, 31-40. https://doi.org/10.1145/3384943.3409420

[23] Zheng, Z., Xie, S., Dai, H., Chen, X., \& Wang, H. (2017). An Overview of Blockchain Technology: Architecture, Consensus, and Future Trends. 2017 IEEE International Congress on Big Data (BigData Congress), 557-564. https://doi.org/10.1109/BigDataCongress.2017.85.

[24] Potharaju, S. P., Sreedevi, M., \& Amiripalli, S. S. (2019). An Ensemble Feature Selection Framework of Sonar Targets Using Symmetrical Uncertainty and Multi-Layer Perceptron (SU-MLP). In Cognitive Informatics and Soft Computing (pp. 247-256). Springer, Singapore.

[25] Wang, J., Wang, S., Guo, J., Du, Y., Cheng, S., \& Li, X. (2019). A Summary of Research on Blockchain in the Field of Intellectual Property. Procedia Computer Science, 147, 191-197. https://doi.org/10.1016/j.procs.2019.01.220

[26] Kitsantas, Thomas \& Vazakidis, Athanasios \& Chytis, Evangelos. (2019). A Review of Blockchain Technology and Its Applications in the Business Environment.

[27] Zhou, L., Zhang, L., Zhao, Y., Zheng, R., \& Song, K. (2020). A scientometric review of blockchain research. Information Systems and E-Business Management. https://doi.org/10.1007/s10257-02000461-9 